\title{
Enterocolitis necrotizante
}

Drs. A. Harún ${ }^{*}$, A. Vásquez ${ }^{*}$, J. Careaga y J.J. Latorre

La enterocolitis necrotizante es una enfermedad de alta mortalidad, descrita clásicamente como propia del RN y que afecta en especial a prematuros y RN de bajo peso, con antecedentes de hipoxia, infección y/o trastornos hemodinámicos, entre otros ${ }^{1-2-4-5-6-14}$.

Clínicamente se caracteriza por retardo en el vaciamiento gástrico, vómitos frecuentes, deposiciones generalmente normales que pueden volverse diarreicas y con sangre, crisis de apnea y cianosis, manifestaciones de pseudo obstrucción intestinal y progresivo compromiso del estado general $^{1-2-45-6-14}$.

En el intestino se encuentran necrosis, hemorragias, a veces perforaciones que pueden causar neumoperitoneo $y / o$ peritonitis, siendo lo más característico la presencia de aire en la pared intestinal (neumatosis intestinal) y a veces en el sistema porta ${ }^{1-2-4-6-6-14}$.

*Hospital Luis Calvo Mackenna.
Esta enfermedad habría sido descrita ya en 1838 por Simpson, quien presentó 25 casos de peritonitis no explicada; pero fue Genersich, en 1891, quien presentó el primer caso de un RN, con el cuadro clínico y anatomopatológico de la EN. Aparece la indicación operatoria con la primera sobrevida en 1943. Numerosas publicaciones aparecen posteriormente relacionando el cuadro con otras patologías abdominales. Sólo en 1964 se la describe como un cuadro con características clínicas, radiológicas y anatomopatológicas bien definidas y desde entonces se proponen múltiples teorías para explicar su etiopatogenia ${ }^{1-2-6-6}$

Recientemente hemos detectado algunos casos de EN en lactantes desnutridos con diarrea aguda con deshidratación, que constituye un material distinto de lo comunicado en la literatura extranjera. El diagnóstico se ha corroborado con radiografía de abdomen simple que demostró neumatosis intestinal y/o gas en el 
sistema porta, o bien con el estudio anatomopatológico. Nuestros pacientes recibieron tratamiento médico exclusivo, a diferencia del material de otras publicaciones que cuentan con un porcentaje elevado de casos tratados quirúrgicamente $e^{1-2-4-5}$.

Nos pareció importante comunicar nuestra experiencia en el manejo de la EN por dos motivos: 1) por ser una entidad que en nuestro mędio es más bien propia del lactante menor desnutrido con diarrea aguda que del RN prematuro y, dada la incidencia de desnutrición y diarrea aguda en nuestro medio, el conocimiento de esta patología adquiere enorme importancia, y 2) la sobrevida de los casos más recientes, obtenida por la pesquisa precoz y dirigida de este síndrome.

\section{MATERIAL}

Se revisan retrospectivamente los casos de EN diagnosticados entre enero de 1970 y junio de 1975 en el Hospital Luis Calvo Mackenna, con un total de 32 casos que se dividen en dos grupos:

1. Un grupo que denominamos de revisión anatomopatológica (RAP), que comprende 21 pacientes del período 1970 a 1974, obtenidos del archivo de A.P. de nuestro hospital, en quienes no se sospecha el diagnóstico en vida.

2. Un grupo que denominamos de revisión clínica ( $R C$ ), que comprende 11 pacientes del período 1974 a 1975, todos diagnosticados precozmente, excepto uno, en quien sólo se sospecha el diagnóstico en vida pero con comprobación A.P. posterior. Se revisan los antecedentes clínicos, exámenes de laboratorio y A.P. de cada paciente, desestimándose aquellos datos sin importancia estadística o no relacionados con la $\mathrm{EN}$.

Se tomaron los parámetros clínicos y de laboratorio en el momento que éstos estaban relacionados con la enfermedad, consignándose por ello los datos de los tres días previos al diagnóstico o fallecimiento.

\section{RESULTADOS}

Se encontró una distribución de la enfermedad semejante en ambos sexos con un predominio de lactantes menores sobre $\mathrm{RN}$, constituyendo los lactantes entre 1 y 3 meses el grupo etario más importante (tablas 1 y 2 ).

El estado nutritivo fue deficiente, hubo un claro predominio de desnutridos sobre eut róficos: 72 y $81 \%$ de desnutridos para los grupos de RAP y RC, respectivamente (tabla 3 ).

Tabla 1 DISTRIBUCION POR EDAD Y SEXO EN 21
PACIENTES CON EN

Revisión anatomopatológica: 1970-1974

Hospital Luis Calvo Mackenna

\begin{tabular}{lrrrrrr}
\hline \multirow{2}{*}{ Edad } & \multicolumn{3}{c}{ Sexo } & & Totales \\
& \multicolumn{2}{c}{ Hombres } & \multicolumn{2}{c}{ Mujeres } & & \\
\hline & $\mathrm{N}$ & $\%$ & $\mathrm{~N}$ & $\%$ & $\mathrm{~N}$ & $\%$ \\
RN & 5 & 24,0 & 0 & 0,0 & 5 & 24,0 \\
1 a 3 ms. & 3 & 14,0 & 6 & 28,8 & 9 & 43,2 \\
3 a 6 ms. & 2 & 9,6 & 4 & 19,2 & 6 & 28,8 \\
+ de 6 ms. & 0 & 0,0 & 1 & 4,8 & 1 & 4,8 \\
\cline { 2 - 8 } Total & 10 & 48,0 & 11 & 52,0 & & \\
\hline
\end{tabular}

Tabla 2

DISTRIBUCION POR EDAD Y SEXO EN 11 PACIENTES CON EN

Revisión clínica: 1974-1975

Hospital Luis Calvo Mackenna

\begin{tabular}{llrrrrr}
\hline \multirow{2}{*}{ Edad } & \multicolumn{3}{c}{ Sexo } & \multicolumn{2}{c}{ Totales } \\
& \multicolumn{2}{c}{ Hombres } & \multicolumn{1}{c}{ Mujeres } & & \\
\hline & $\mathrm{N}$ & $\%$ & $\mathrm{~N}$ & $\%$ & $\mathrm{~N}$ & $\%$ \\
$\mathrm{RN}$ & 1 & 9,0 & 1 & 9,0 & 2 & 18,0 \\
1 a 3 ms. & 2 & 18,0 & 3 & 27,0 & 5 & 45,0 \\
3 a 6 ms. & 1 & 9,0 & 2 & 18,0 & 3 & 27,0 \\
+ de 6 ms. & 1 & 9,0 & 0 & 0,0 & 1 & 9,0 \\
\cline { 2 - 7 } Total & 5 & 45,0 & 6 & 54,0 & & \\
\hline
\end{tabular}

Tabla 3

ESTADO NUTRITIVO EN PACIENTES CON EN

Revisión anatomopatológica : 21 (1970-1974)

Revisión clínica : 11 (1974-1975)

Hospital Luis Calvo Mackenna

\begin{tabular}{llrrr}
\hline Estado nutritivo & \multicolumn{2}{c}{$R C$} & \multicolumn{2}{c}{$R A P$} \\
\hline & $\mathrm{N}$ & $\%$ & $\mathrm{~N}$ & \multicolumn{1}{c}{$\%$} \\
Eutróficos & 1 & 9,0 & 4 & 19,2 \\
Desnutridos Grado 1 & 3 & 27,0 & 2 & 9,6 \\
Desnutridos Grado 2 & 3 & 27,0 & 4 & 19,2 \\
Desnutridos Grado 3 & 3 & 27,0 & 9 & 43,2 \\
No clasificable & 1 & 9,0 & 2 & 9,6 \\
\hline
\end{tabular}


Aunque el peso de nacimiento es de importancia secundaria en los lactantes con EN, la mitad de los pacientes pesó al nacer $2.500 \mathrm{gr}$ o menos, pero en el grupo de los $\mathrm{RN}$, de un total de 7,5 pesaron menos de $2.500 \mathrm{gr}$, otro más de 2.500 gr y uno en quien no se consignó el peso de nacimiento.

En relación con la alimentación recibida al iniciarse la enfermedad, se obtuvieron antecedentes en 24 de los 32 pacientes, de los cuales 21 eran alimentados artificialmente $(65 \%)$ a su ingreso al hospital y 3 recibían alimentación materna exclusiva $(9,37 \%)$.

En el análisis del cuadro clínico se encontró que 20 de los 32 pacientes $(62,5 \%)$ ingresó al hospital con un cuadro de diarrea aguda; de los 12 restantes, 9 pacientes $(28,13 \%)$ lo desarrollaron durante su hospitalización y sólo 3 no lo presentan $(9,37 \%)$. Durante la evolución de la diarrea puede aparecer sangre en las deposiciones, hecho más frecuente en el grupo de RAP (tabla 5).

Los vómitos aparecen en un alto porcentaje de los pacientes, pero fue mayor en el grupo de $\mathrm{RC}$ en relación al de $\mathrm{RAP}$, diferencia para la cual no encontramos explicación satisfactoria. Estos pueden ser biliosos o de retención, aproximadamente en un tercio de los casos (tabla 5).

La distensión abdominal fue también frecuente, presentándose con diferentes grados de intensidad y en un número importante fue progresiva (tabla 6 ).

Todos los pacientes presentaron deshidratación en un momento de la evolución, si bien en el período considerado para el recuento de datos, el porcentaje de deshidratación fue menor, ya que en un pequeño porcentaje se había corregido previamente en forma parenteral (tabla 6).

La acidosis se encontró en el $100 \%$ de los pacientes del grupo de RC y sólo en el $43 \%$ del RAP, lo que se explica por el mejor seguimiento de los pacientes del primer, grupo mencionado (tabla 6).

En relación al tránsito intestinal, un tercio de los pacientes presentó un cuadro de pseudoobstrucción intestinal, caracterizado por gran distensión abdominal y detención del tránsito; 3 pacientes del grupo de RAP fueron sometidos a intervención quirúrgica por esta complicación, pensando en un cuadro obstructivo de otra causa (tabla 6).

\section{Tabla 4}

PESO DE NACIMIENTO EN PACIENTES CON EN

Revisión anatomopatológica : 21 (1970-1974)

Revisión clínica : 11 (1974-1975)

Hospital Luis Calvo Mackenna

\begin{tabular}{lcccc}
\hline Peso de nacimiento & & $R C$ & \multicolumn{2}{c}{$R A P$} \\
\hline & $\mathrm{N}$ & $\%$ & $\mathrm{~N}$ & $\%$ \\
$-\mathrm{de} 2.500 \mathrm{grs}$ & 4 & 36,0 & 10 & 48,0 \\
+ de $2.500 \mathrm{grs}$ & 6 & 54,0 & 11 & 52,0 \\
\hline
\end{tabular}

Tabla 5

\section{DIARREA Y VOMITOS EN PACIENTES CON EN}

Revisión anatomopatológica : 21 (1970-1974)
Revisión clínica
Hospital Luis Calvo Mackenna

\begin{tabular}{lrrrr}
\hline & \multicolumn{2}{c}{$R C$} & \multicolumn{2}{c}{$R A P$} \\
\cline { 2 - 5 } & $\mathrm{V}$ & $\%$ & $\mathrm{~N}$ & $\%$ \\
Diarrea & 10 & 90,0 & 19 & 91,2 \\
$\quad$ Sin sangre & 8 & 72,0 & 10 & 48,0 \\
$\quad$ Con sangre & 2 & 18,0 & 9 & 43,2 \\
Vómitos & 9 & 81,0 & 10 & 48,0 \\
$\quad$ Sin caract. especial & 5 & 45,0 & 4 & 19,2 \\
Biliosos o retención & 4 & 36,0 & 6 & 28,8 \\
\hline
\end{tabular}

Tabla 6

IDISTENSION ABDOMINAL, DESHIDRATACION, ACIDOSIS Y CLADRO DE PSEUDO OBSTRUCCION EN PACIENTES CON EN

Revisión anatomopatológica : 21 (1970-1974)

Revisión clínica : 11 (1974-1975)

Hospital Luis Caivo Mackenna

\begin{tabular}{lrrrr}
\hline & \multicolumn{2}{c}{$R C$} & \multicolumn{2}{c}{$R A P$} \\
\cline { 2 - 5 } & $\mathrm{N}$ & $\%$ & $\mathrm{~N}$ & $\%$ \\
Distensión abdominal & 10 & 90,0 & 16 & 76,8 \\
$\quad$ Evolución progresiva & 5 & 45,0 & 12 & 57,6 \\
$\quad$ Evolución uniforme & 5 & 45,0 & 5 & 19,2 \\
Deshidratación & 8 & 72.0 & 18 & 86,4 \\
Acidosis & 11 & 100,0 & 9 & 43,2 \\
Pseudo obstrucción & & & & \\
intestinal & 3 & 27,0 & 8 & 38,4 \\
\hline
\end{tabular}

De los exámenes de laboratorio sólo se analizan dos: radiografía de abdomen simple y hemocultivos. La radiografía de abdomen simple se obtuvo en todos los pacientes del grupo de RC, con un $90 \%$ de positividad para la neumatosis intestinal y en uno se encontró gas en el sistema porta. En cambio en los 4 pacientes del grupo de RAP estudiados con radiografía de abdomen, 
sólo se encontró distensión de asas (tabla 7). Los hemocultivos en ambos grupos revelan a la Klebsiella enterobacter como germen más frecuente, aunque de escasa incidencia (tabla 8 ).

Los hallazgos de AP muestran como hechos más frecuentes la necrosis, hemorragia, inflamación y neumatosis intestinal (tabla 9).

Tabla 7

\section{RADIOGRAFIA DE ABDOMEN SIMPLE EN PACIENTES CON E.N.}

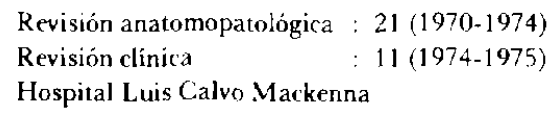

\begin{tabular}{lrrrr}
\hline Rx de abdomen stmple & \multicolumn{2}{c}{$R C$} & \multicolumn{2}{l}{$R A P$} \\
\hline & $\mathrm{N}$ & $\%$ & $\mathrm{~N}$ & $\%$ \\
& & & & \\
Total Rx & 11 & 100 & 4 & 19,2 \\
$\quad$ Neumatosis & 10 & 90 & 0 & 0,0 \\
Gas en sist. porta & 1 & 9 & 0 & 0,0 \\
Distensión de asas & 11 & 100 & 4 & 100,0 \\
\hline
\end{tabular}

Tabla 8

HEMOCULTIVOS EN PACIENTES CON EN

$\begin{array}{ll}\text { Revisión anatomopatológica : } & 21 \text { (1970-1974) } \\ \text { Revisión clínica } & : 11 \text { (1974-1975) }\end{array}$

Hospital Luis Calvo Mackenna

\begin{tabular}{lcccc}
\hline Hemocultivos & \multicolumn{2}{c}{$R C$} & \multicolumn{2}{c}{$R A P$} \\
\hline Total de exámenes & $\mathrm{N}$ & $\%$ & $\mathrm{~N}$ & $\%$ \\
Positivos & 8 & 72,0 & 7 & 33,6 \\
$\quad$ Klebsiella & 1 & 9,0 & 4 & 19,2 \\
Streptococcus & 1 & 9,0 & 2 & 9,6 \\
$\quad$ Pseudomona & 0 & 0,0 & 1 & 4,8 \\
Negativos & 0 & 0,0 & 1 & 4,8 \\
\hline
\end{tabular}

Tabla 9

HALLAZGOS ANATOMOPATOLOGICOS EN 21 PACIENTES CON EN

Revisión anatomopatológica : 21 (1970-1974)

Hospital Luis Calvo Mackenna

\begin{tabular}{lcr}
\hline Hallazgos de AP & $N$ & $\%$ \\
\hline Neumatosis & 12 & 57,6 \\
Inflamación & 20 & 96,0 \\
Hemorragia & 17 & 81,6 \\
Necrosis & 21 & 100,0 \\
Perforación & 3 & 14,4 \\
Peritonitis & & 33,6 \\
\hline
\end{tabular}

Otros antecedentes como hipoxia, CID, shock y exámenes de laboratorio como hemograma, orina completa, coprocultivos, $\mathrm{pH}$ y Benedict en deposiciones, etc., fueron considerados pero se descartaron por no ser de importancia estadística o no atribuibles a la EN como ya se mencionó.

\section{DISCUSION}

Del análisis de los resultados, vemos que la EN en nuestro medio predomina en lactantes menores con un cuadro de diarrea aguda con deshidratación y acidosis como base, a diferencia de la mayoría de lo encontrado en la literatura extranjera, coincidiendo sólo con trabajos latinoamericanos $^{6-13}$.

Para el análisis de los casos, los dividimos en dos grupos ya que reflejan dos etapas en el diagnóstico, tratamiento y pronóstico de nuestros pacientes:

Una primera etapa en que no hubo diagnóstico clínico y, por lo tanto, la enfermedad cursó espontáneamente hasta su fal'ecimiento, por lo que se encuentran hechos que implican etapas más avanzadas de la enfermedad, como son un mayor porcentaje de deposiciones con sangre, vómitos de carácter bilioso o de retención y mayor positividad de los hemocultivos.

En la etapa actual, en que hubo sospecha y comprobación precoz del diagnóstico, se llevó a cabo un seguimiento más estricto tanto clínico como de laboratorio, con la realización de medidas terapéuticas adecuadas, lo que permitió elevar la sobrevida de 0 a $72 \%$.

En cuanto a la etiopatogenia de la EN, ésta es desconocida. Son numerosas las hipótesis que se han postulado para explicarla. Se han considerado factores tan diversos como: mecánicos, amnionitis, fenómeno de Schwartzmann localizado, endotoxinas, isquemia intestinal, déficit de lisosimas, cateterización de vasos umbilicales, malabsorción de hidratos de carbono, alimentación hiperosmolar, CID, viremias, bacteremias, ingestión de líquido amniótico infectado, intolerancia a disacáridos con hipopotasemia, etc. La mayor parte de ellos se ha ido descartando por no haberse logrado comprobación clínica ni experimental ${ }^{3-4-5-13}$.

Interesantes y recientes investigaciones, con una casuística de más de 100 casos entre ambos 
trabajos, han permitido plantear un mecanismo de producción de la enfermedad que incluye sólo los factores que en alguna forma han tenido comprobación clínica o experimental. Estos trabajos fueron realizados en un material en que todos eran $\mathrm{RN}$, con un predominio de $\mathrm{RN}$ de pretérmino (78 a 90\%) y con una edad pro- medio de presentación de la enfermedad de 6,5 $\operatorname{dias}^{4-5}$

Esta teoría propone la existencia de al menos 3 factores etiopatogénicos fundamentales en la producción de la EN: 1) injuria intestinal, 2) presencia de bacterias, 3) presencia de un factor metabólico (gráfico 1).

\section{Gráfico 1}

\section{PATOGENIA DE LA ENTEROCOLITIS NECROTIZANTE}

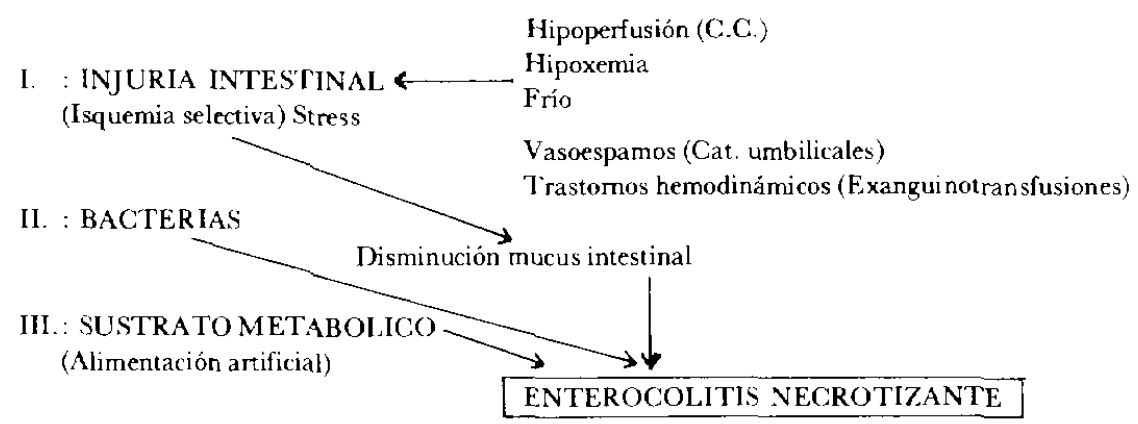

Se sabe que el stress produce una redistribución de la sangre fuera de aquellos territorios que toleran mejor la isquemia, dentro de los cuales se encuentra la red mesentérica. El stress de los RN puede estar causado por factores como hipoxia, frio, hipoperfusión en cardiopatías congénitas, vasoespasmos en catéteres umbilicales, trastornos hemodinámicos en las exanguinotransfusiones, entre otros, y al producir una redistribución sanguínea provocaría isquemia selectiva a nivel intestinal, ocasionando una disminución en la producción de mucus, y al ser eliminado este factor protector de la mucosa permitiría la acción enzimática produciendo daño tisular. La mucosa dañada permitiría la invasión de microorganismos con proliferación de ellos, facilitada esta última por el factor metabólico que corresponde a la alimentación. En cuanto a la alimentación, además de su presencia es importante la calidad de ella, ya que la alimentación artificial se encuentra desprovista de los factores protectores de la leche materna, principalmente macrófagos e Iga., además si la alimentación es hiperosmolar favorece aún más la producción de la enfermedad por daño tisular directo $^{3}$ 4-5-6.7 8-9-10-11-12-14.

En nuestros pacientes se encuentran los factores mencionados: la injuria intestinal estaría condicionada por la hipoxia consecutiva a la hipoperfusión enteral ocasionada por la deshidratación presente en todos los pacientes y agravada en aquéllos en que hubo acidosis metabólica; en cuanto a la presencia de bacterias en el intestino, es altamente probable la existencia de gérmenes patógenos en lactantes desnutridos que presentaron diarrea aguda, aun cuando en nuestro material los coprocultivos fueron negativos, sin perjuicio de considerar la flora intestinal aerobia y anaerobia habitual.

Por último, casi todos nuestros pacientes recibían alimentación artificial y por el hecho de ser desnutridos en los que sólo un escaso porcentaje había recibido alimentación matema las primeras semanas de vida, estarían dadas las condiciones de déficit inmunológico por una parte y el aporte del sustrato metabólico para el desarrollo bacteriano, por otra.

Finalmente nos referiremos al tratamiento:

En el grupo de RAP los pacientes fueron tratados como cuadros de diarrea aguda con deshidratación y acidosis, no pudiendo ser encuadrados en un esquema bien determinado.

En el grupo de RC, en cambio, se tratan en forma dirigida, evolucionándose progresivamente a un esquema de tratamiento bien determinado, no siendo necesaria la cirugía en nin- 
gún caso. Por la experiencia así obtenida, se ha iniciado una nueva etapa en el diagnóstico y en el tratamiento de la EN.

En esta forma, ante un RN o lactante menor con el cuadro descrito, se inicia un seguimiento clínico y de laboratorio más acucioso, considerando como básico los siguientes exámenes: hemograma, hemocultivos, ionogramas seriados, reacción de Weber, coprocultivos, $\mathrm{pH}$ y Benedict en deposiciones seriados y radiografia de abdomen simple.

Al ser confirmado el diagnóstico se inicia el siguiente tratamiento:

1. Reposo digestivo con régimen 0 por un período de 6 a 10 dias, dependiendo de la evolución.

2. Aspiración gástrica por un período de acuerdo a la evolución del cuadro.

3. Hidratación parenteral con corrección de los trastornos hidroelectrolíticos de acuerdo al ionograma (Promedio $160 \mathrm{ml} / \mathrm{kgr}$ ).

4. Plasmoterapia a dosis de $40 \mathrm{ml} / \mathrm{kgr} /$ día como única fuente de proteínas.

5. Vitaminas C y complejo B endovenoso de acuerdo a los requerimientos diarios.

6. Antibióticos: gentamicina (5 a $7 \mathrm{mgr} / \mathrm{kgr} /$ día) y penicilina sódica $(100.000 \mathrm{U} / \mathrm{kgr} /$ día $)$.

7. Transfusión de sangre, en caso necesario de acuerdo al Hto. y $\mathrm{Hb}$.

8. Realimentación con control previo de radiografía de abdomen simple para verificar la desaparición de la neumatosis intestinal y reacción de Weber para certificar la ausencia de sangramiento.

Con este tratamiento se ha obtenido una sobrevida de $72 \%$, no siendo necesaria la cirugia, por lo que se propone como tratamiento, ya que la alimentación parenteral es un método aún restringido en nuestro medio.

Por lo dicho, no estamos excluyendo a la cirugía como parte del tratamiento del paciente con EN en un momento dado de la evolución de la enfermedad, sino ésta debe tenerse siempre presente frente a un enfermo con esta patologia. Al revisar la literatura, la cirugía se lleva a cabo en un alto porcentaje de estos pacientes (40 a $60 \%$ ); en cuanto al momento en que ésta debe indicarse no hay acuerdo unánime, excepto frente a la presencia de peritonitis o neumoperitoneo, pero la dificultad se encuentra en las etapas previas, indicándose por algunos autores frente al empeoramiento del estado general, presencia de acidosis y/o shock, otros ante la confirmación de CID o presencia de líquido peritoneal, etcé$\operatorname{tera}^{1 \cdot 2 \cdot 4 \cdot 6 \cdot 14}$

\section{RESUMEN}

Se presentan 32 casos de enterocolitis necrotizante que corresponden a material clínico y de anatomia patológica del Hospital Luis Calvo Mackenna, entre 1970 y 1975 . Se observan dos etapas claras en cuanto a diagnóstico y tratamiento con un notable aumento de la sobrevida de 0 a $72 \%$ durante el ultimo año.

Se discute una teoría que pretende explicar la patogenia de esta entidad clínica.

Se propone un esquema terapéutico posible de realizar en nuestro medio, considerando siempre la indicación quirúrgica en un momento dado de la evolución.

\section{SUMMARY}

Summarized are 32 patients with necrotizing enterocolitis collected from the Inpatient and Pathology Services of the Calvo Mackenna Hospital between 1970 and 1975. Analisis of the cases shows two clear pronostic differences with diminished mortality in the year 1975. (From 100\% to $28 \%$ ).

An hypothesis is proposed that explains the pathogenesis of this clinical entity.

A treatment plan that can be accomplished with our limited facilities is proposed, always considering sugery as a possible therapeutic resource.

\section{BIBLIOGRAFIA}

Stevenson, J.K.; Oliver, T.K.; Graham, B. et al. J. Pediatr. Surg., 6:28, 1971.

2 Touioukian, R.S.; Berdon. W.E.; Amoury, R.A. et al. J. Pediatr. Surg., 2:388, 1967

i Barlow, B; Santulli, T.V.; Hetrd, W.C.. et al. J. Pediatr. Surg., 9:587, 1974

- Frantz, I.D.; L'Heureux, P.; Engel, R.R. et. al. J. Pediatr., 86:259, 1975.

5 Santulli, T:V.; Schullinger, J.A.; Hetrd, W.C. et. al. in Pediatr., 55:376, 1975

- Núñez, R. y Nuraimbell, F. Rev. Méd. Hosp. Nal. Niños Dr. Carlos Sáenz Herrera, 9(1):1, 1974.

; Book, L.S.; Herbst, J.J.; Jung, A.L. et al. Pediatr., $87: 602,1975$

8 Vimig, N.L.; Reynolds, J.W. Am. J. Dis. Child., 128: 186,1974

- De Lemos, R.A.; Rogers, f.; Mac Laughin, G.W. Pediatr. Res., 8:380, 1974.

10 Polin, R.; Pollack, P.F.; Barlow, B. et al. Pediatr. Res., $8: 384,1974$

1 Pitt, J.; Barlow, B.; Heird, W.; Santulli, T. Pediatr. Res., 8:384, 1974.

12 Krouskop, R.; Brown, E. and Sweet, A. Pediatr. Res., $8: 383,1974$

13 Coello-Ramírez, P.; Gutiérrez-Topele, $G$. and Lifshitz, F. Am.J. Iis. Child., 120:3, 1970.

14 Proceedings of the Ross Conference on Pediatric Research. June 1975. 